\title{
Corpus
}

\section{Les mots du vin : experts et novices diffèrent-ils quand ils décrivent des vins?}

Dominique Valentin, Sylvie Chollet et Hervé Abdi

\section{(2) OpenEdition \\ 1 Journals}

\section{Édition électronique}

URL : http://journals.openedition.org/corpus/36

DOI : $10.4000 /$ corpus.36

ISSN : 1765-3126

\section{Éditeur}

Bases; corpus et langage - UMR 6039

\section{Édition imprimée}

Date de publication : 15 décembre 2003

ISSN : 1638-9808

\section{Référence électronique}

Dominique Valentin, Sylvie Chollet et Hervé Abdi, «Les mots du vin : experts et novices diffèrent-ils quand ils décrivent des vins ?», Corpus [En ligne], 2 | 2003, mis en ligne le 15 décembre 2004, consulté le 08 septembre 2020. URL : http://journals.openedition.org/corpus/36 ; DOI : https://doi.org/ 10.4000 /corpus.36

Ce document a été généré automatiquement le 8 septembre 2020.

(c) Tous droits réservés 


\title{
Les mots du vin : experts et novices diffèrent-ils quand ils décrivent des vins?
}

\author{
Dominique Valentin, Sylvie Chollet et Hervé Abdi
}

Introduction

1 Qu'est ce qu'un expert en vin ? En quoi un expert en vin diffère-t-il d'un novice ? Gawel (1997) fait, à juste titre, remarquer que l'entrainement et l'expérience sont des concepts qui n'ont pas été clairement définis et que leurs limites ne sont pas bien établies. Selon le dictionnaire Larousse de la langue française, un expert est «un spécialiste chargé d'apprécier, de vérifier ", et selon le dictionnaire des sciences cognitives, «l'expertise s'acquière progressivement après de nombreuses années d'études et de pratique » et est «limitée à un domaine particulier ». Dans le cas de l'expertise en vin, le domaine particulier est celui de la dégustation de vins. Un expert en vins est donc une personne qui a appris à déguster les vins. Selon Peynaud et Blouin (1996) l'action de déguster renvoie à la notion de «goûter avec attention un produit dont on veut apprécier la qualité ». Déguster un vin c'est « le soumettre à nos sens, en particulier ceux du goût et de l'odorat. C'est essayer de le connaître en recherchant ses différents défauts et ses différentes qualités et en les exprimant ». En résumé, un expert en vins se caractérise donc par sa connaissance des vins, connaissance acquise à la suite de nombreuses dégustations, et par sa capacité à décrire des vins. Nous nous intéresserons ici à cette capacité. Plus particulièrement, nous allons chercher à comparer des descriptions de vins effectuées par des experts avec celles réalisées par des novices : les descriptions des experts sont-elles plus riches et ont-elles une valeur communicative plus forte que celle de novices?

2 Le débat sur la valeur communicative des descriptions de vins fournies par des experts a été ouvert par Lehrer (1975). Utilisant une tâche d'appariement (i.e., une tâche où l'on demande à des sujets de reconnaître des vins à partir de leurs descriptions), Lehrer a montré que la capacité à communiquer sur les vins ne semble pas être facilement acquise par les experts. Un peu plus tard, Lawless (1984) rapporte que si les 
descriptions d'experts conduisent effectivement à des performances d'appariement décevantes, elles semblent toutefois avoir une plus grande valeur communicative que celles de novices (2,58 appariements corrects sur 6 descriptions pour les experts contre 1,81 pour les novices). Cette supériorité des experts a été retrouvée par Solomon (1990). Une analyse des termes utilisés par les experts et les novices suggère que l'avantage des experts serait dû au vocabulaire employé : les experts emploient des termes plus précis que les novices dans leurs descriptions. En accord avec cette interprétation Gawel (1997) montre que la performance de novices est significativement meilleure lorsqu'ils doivent retrouver des vins à partir de la description d'un expert qu'à partir de celle d'un novice. Finalement, Chollet et Valentin (2000) indiquent que les descriptions réalisées par des experts comportent davantage de termes techniques et précis alors que celles des novices comportent davantage de termes globaux. Cette tendance suggère que les experts tendent à percevoir les vins de façon analytique alors que les novices les perçoivent de façon plus holistique.

3 L'objectif de l'étude présentée ici est d'examiner de plus près cette hypothèse. Précisément, nous avons demandé à deux groupes de sujets (un groupe d'experts et un groupe de novices) de décrire une série de vins puis de réaliser une tâche d'appariement à partir des descriptions ${ }^{1}$. Contrairement aux études précédentes (Lehrer, 1975 ; Lawless, 1984 ; Solomon, 1990, Gawel, 1997), les descriptions effectuées n'étaient pas des descriptions libres mais des descriptions à choix forcé réalisées à partir d'une liste de descripteurs dérivée de «la roue des vins» (Wine Aroma Wheel Copyright $@ 1990$, A.C. Noble ; voir figure 1 ci-dessous).

4 La roue des vins rassemble des termes décrivant les arômes du vin. Ces termes sont disposés en trois rangées qui vont du plus général (au centre) au plus spécifique (en bordure, e.g., fruité > agrume > citron).

5 Notre hypothèse, en utilisant ce type de liste de termes, est que les experts choisiraient plutôt des termes de la partie externe de la roue (i.e., des termes plus spécifiques) alors que les novices utiliseraient plutôt des termes de la partie centrale (i.e., des termes plus généraux). Les termes globaux étant moins informatifs que les termes spécifiques, les descriptions des novices devraient conduire à de plus faibles performances d'appariement que les descriptions des experts. Nous avons ensuite analysé les descriptions obtenues pour chaque niveau de catégorisation à l'aide d'analyses factorielles des correspondances (AFC, Benzécri et Benzécri, 1980). Notre hypothèse était que la similarité (mesurée à l'aide d'une distance procrustéenne) entre les cartes factorielles obtenues pour les experts et pour les novices diminuerait avec le niveau de spécificité des termes. 


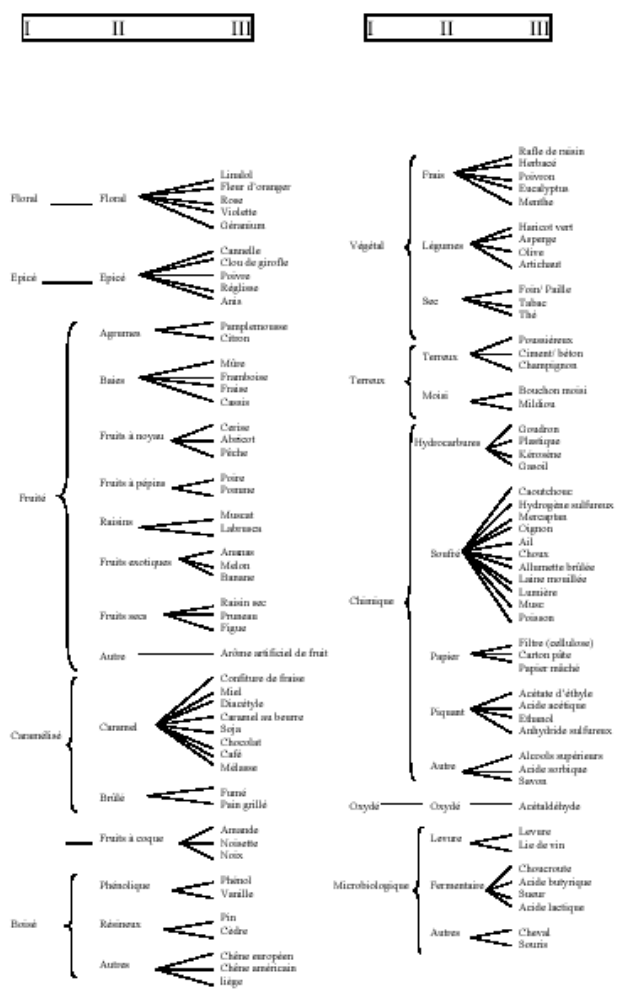

Figure 1 : Liste de termes dérivée de Noble (1990)

1. Matériel et méthode

6 1.1. Participants. Deux groupes de sujets ont participé à cette étude. Le premier groupe (appelé « experts ») était composé de 20 étudiants âgés de 21 à 27 ans (4 femmes et 16 hommes) en fin de deuxième année de préparation du diplôme national d'œnologie de l'I.U.V.V. (Institut Universitaire de la Vigne et du Vin) à Dijon. Le deuxième groupe (appelé «novices») était composé de 19 étudiants novices âgés de 18 à 25 ans ( 9 femmes et 10 hommes) recrutés sur la base du volontariat au sein du campus dijonnais. Parmi les (nombreux) candidats intéressés par cette étude, nous avons sélectionné, à l'aide d'un questionnaire, les personnes n'ayant aucune connaissance particulière dans le domaine du vin.

7 1.2. Stimuli. Nous avons sélectionné six paires de vins rouges à partir de 27 vins rouges français d'Appellation d'Origine Contrôlée (A.O.C.) et d'un vin américain (californien). Les vins français étaient issus de sept grandes régions viticoles qui sont le Beaujolais, le Bordelais, la Bourgogne, les Côtes du Rhône, le Languedoc-Roussillon, le Sud-Ouest et le Val de Loire. Pour réaliser cette sélection, nous avons demandé aux sujets experts et novices d'effectuer une tâche de "tri-libre ». Les sujets devaient sentir les 28 vins, puis les regrouper en fonction de leur odeur en mettant ensemble ceux qui, pour eux, se ressemblaient. Les résultats ont été compilés sous forme de matrices de co-occurrences et soumis à une analyse de similitude (MDS). A partir de ces résultats nous avons choisi six paires de vins, chacune constituée de deux vins les plus différents possibles ( $c f$. tableau I). Le premier vin de chaque paire correspondait à la cible et le second au distracteur lors de la tâche d'appariement.

8 Les vins étaient évalués exclusivement par la technique de flairage. Les échantillons (30 ml) étaient présentés dans des verres à vin « INAO » recouverts d'une boîte de Pétri et 
sous lumière rouge pour éviter que la couleur des vins ne soit prise en compte par les sujets. Les verres étaient servis à température ambiante une demi-heure avant le début de l'épreuve.

Tableau I : Paires de vins utilisées dans la tâche d'appariement

\begin{tabular}{|c|l|l|}
\hline Paire & Cibles & Distracteurs \\
\hline 1 & $\begin{array}{l}\text { Zinfandel, } \\
\text { (Californie) }\end{array}$ & $\begin{array}{l}\text { Macon rouge, 1997 } \\
\text { (Bourgogne) }\end{array}$ \\
\hline 2 & $\begin{array}{l}\text { Marcillac, 1998 (Sud- } \\
\text { Ouest) }\end{array}$ & $\begin{array}{l}\text { Côte de Provence, 1998 } \\
\text { (Languedoc-Roussillon) }\end{array}$ \\
\hline 3 & $\begin{array}{l}\text { Côtes du Rhône domaine } \\
\text { Beauplan, 1998 }\end{array}$ & Beaujolais village, 1998 \\
\hline 4 & $\begin{array}{l}\text { Buzet, 1998 (Sud Ouest) } \\
\text { Beraine Amboise, 1998 } \\
\text { (Bal de Loire) }\end{array}$ \\
\hline 6 & $\begin{array}{l}\text { Costière de Nìmes,1998 } \\
\text { (Languedoc-Roussillon) }\end{array}$ & $\begin{array}{l}\text { Château de Beaumont, } \\
1998 \text { (Bordeaux) }\end{array}$ \\
\hline
\end{tabular}

1.3. Procédure. Dans un premier temps, les sujets étaient invités à décrire les 12 vins à l'aide de la liste de descripteurs présentée dans la figure 1. Pour chaque échantillon, les sujets devaient entourer les descripteurs qui leur semblaient les plus appropriés pour décrire leurs impressions. Ils pouvaient entourer autant de mots qu'ils le souhaitaient et les choisir dans n'importe quelle catégorie. Dans un deuxième temps, les sujets devaient réaliser une épreuve d'appariement à choix forcé. Parmi les 12 descriptions réalisées précédemment, seules les six descriptions correspondant aux vins cibles (tableau 1) étaient présentées aux sujets. Pour éviter une familiarisation avec ces six vins lors de la phase de description, les sujets décrivaient également les six vins distracteurs (les descriptions correspondant aux vins distracteurs n'étaient pas utilisées dans la tâche d'appariement). Chacune des descriptions présentées était accompagnée de deux verres de vin: le verre cible et le verre distracteur. La tâche consistait donc à retrouver le verre correspondant à la description. Chaque groupe de sujets (experts et novices) était divisé en deux sous-groupes: l'un recevait les descriptions élaborées par les novices et l'autre celles des experts. Un sujet ne recevait jamais sa propre description.

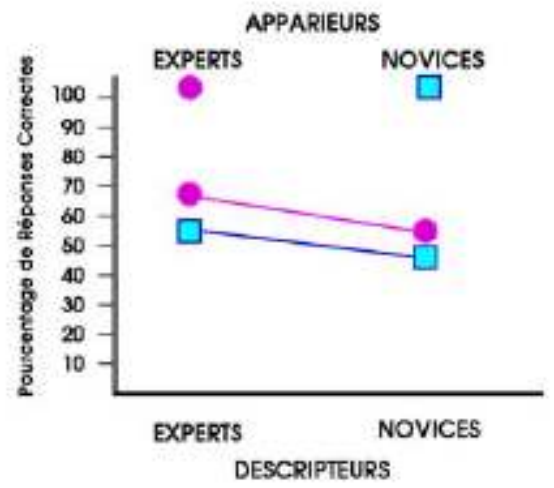

Figure 2: Performance d'appariement des experts et des novices

2. Résultats2.1. Effet de l'expertise sur la proportion d'appariement correct. 
10 La figure 2 présente la proportion d'appariements corrects obtenus dans les quatre conditions expérimentales. Un appariement est considéré comme correct quand le sujet choisit le vin cible. Un test du $t$ de Student $(p<.05)$ montre que seuls les experts recevant des descriptions d'experts ont une performance d'appariement supérieure au hasard. En d'autres termes, les experts sont plus performants dans une tâche d'appariement que les novices, mais uniquement lorsqu'ils reçoivent des descriptions d'experts. On peut également noter que les novices obtiennent de meilleures performances lorsqu'ils reçoivent des descriptions d'experts que des descriptions de novices. Leurs performances, toutefois ne dépassent pas significativement le niveau du hasard. Les descriptions des experts semblent donc plus informatives que celles des novices.

2.2. Répartition des termes en fonction de leur degré de spécificité.

11 Pour comprendre en quoi les descriptions des experts sont plus efficaces que celles des novices dans une tâche d'appariement nous avons regardé la répartition des termes utilisés par les experts et les novices en fonction de leur niveau de spécificité (voir figure 3 ci-dessous).

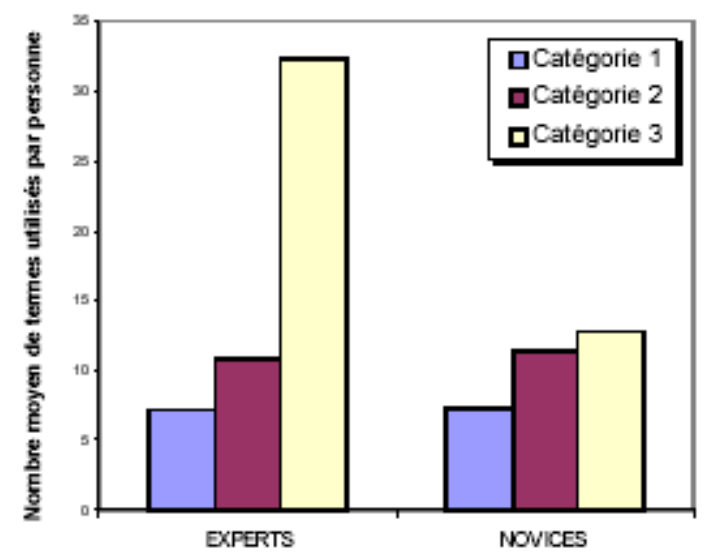

Figure 3 : répartition des termes des experts et des novices en fonction de leur degré de précision

Globalement les experts utilisent plus de termes que les novices et cette différence entre les deux groupes de sujets se fait essentiellement au niveau des termes précis. En effet, experts et novices utilisent un nombre équivalent de termes de niveau I et II, mais les experts utilisent un nombre plus important de termes de niveau III.

2.3. Comparaison des descriptions d'experts et de novices.

13 Pour comparer plus précisément les descriptions des experts et des novices, nous avons réalisé des analyses factorielles des correspondances par niveau de catégorisation et pour chaque niveau nous avons calculé la distance procrustéenne entre les représentations des vins obtenues pour les experts et les novices. La distance procrustéenne entre deux configurations de points est égale à la somme des distances au carré entre les points des deux configurations normalisées après translation, rotation et dilatation d'une des deux configurations (Gower, 1971). Cette transformation, appelée " analyse procrustéenne » a pour but de rendre la deuxième configuration aussi proche que possible de la première configuration. Une petite distance procrustéenne entre deux configurations indique que ces deux configurations se ressemblent. 
Pour le niveau III de catégorisation (i.e., le plus spécifique), nous avons compté le nombre de sujets d'un groupe donné ayant choisi chacun des termes correspondant à ce niveau pour chaque vin. Les termes utilisés par un seul sujet seulement ont ensuite été éliminés de l'analyse. Nous avons ainsi obtenu un tableau de contingence comportant 6 vins et 64 termes pour les experts et un de 6 vins et 26 termes pour les novices. Pour le niveau II de catégorisation, nous avons compté les termes appartenant à ce niveau de catégorisation et ceux appartenant au niveau III de catégorisation correspondant. En effet, par exemple, si le terme «pamplemousse » apparaissait dans une description, il était comptabilisé comme une occurrence dans la catégorie « agrume ». Nous avons ainsi obtenu pour ce niveau de catégorisation deux tableaux de contingence comportant 6 vins et 26 termes, un pour les experts et un pour les novices. Pour le niveau I de catégorisation (i.e., le plus général) nous avons regroupé les termes appartenant aux niveaux II et III de catégorisation et ceux appartenant au niveau I de catégorisation. Nous avons ainsi obtenu pour ce dernier niveau de catégorisation deux tableaux de contingence comportant 6 vins et 11 termes, un pour les experts et un pour les novices. Par souci de simplicité nous n'avons conservé que les deux premières dimensions de chacune des AFC réalisées. Les pourcentages de variance expliquée par ces sous-plans sont présentés dans le tableau II :

Tableau II : Pourcentage de variance expliquée par les deux premières dimensions des AFC réalisées à chaque niveau de catégorisation pour les experts et les novices.

\begin{tabular}{|l|l|l|l|l|l|l|}
\hline & \multicolumn{3}{|c|}{ Experts } & \multicolumn{3}{c|}{ Novices } \\
\hline Niveau & I & II & III & I & II & III \\
\hline Dim. I & 41,92 & 33,23 & 30,83 & 57,61 & 42,02 & 31,12 \\
& & & & & & \\
\hline Dim. 2 & 33,75 & 23,95 & 23,89 & 17,03 & 22,45 & 26,76 \\
\hline Somme & 75,67 & 57,18 & 54,72 & 74,64 & 64,47 & 57,88 \\
\hline
\end{tabular}

Conformément à notre hypothèse, les distances procrustéennes calculées entre les configurations bi-dimensionnelles ainsi obtenues pour les experts et les novices augmentent avec le niveau de catégorisation (niveau III, $d=0,35$; niveau II, $d=0,64$; niveau I, $d=1,27$ ). Les différences entre les configurations obtenues pour les experts et les novices peuvent se voir sur les figures 4,5 et 6 . 


\section{A : Experts - Niveau de catégorisation I}
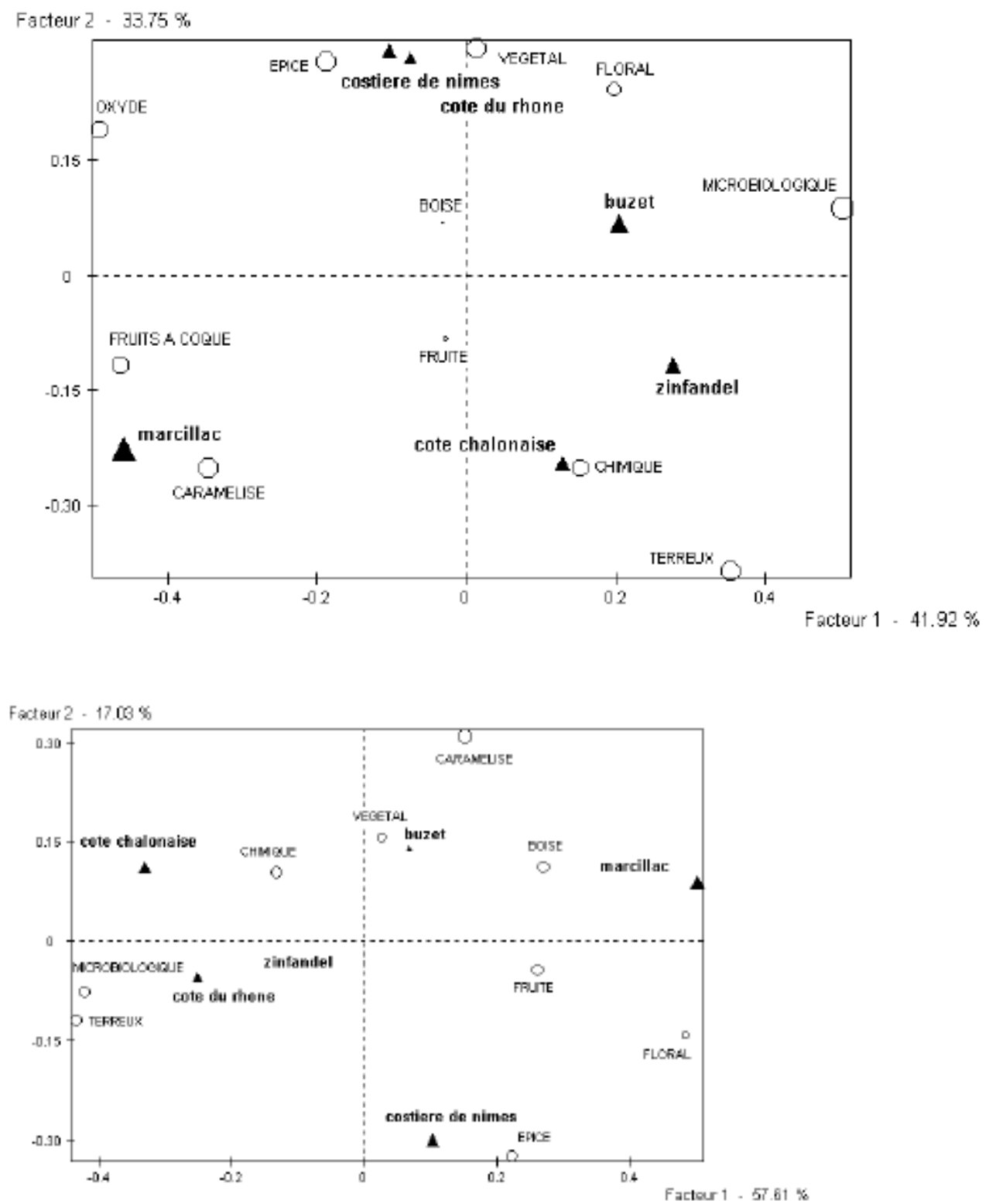

Figure 4 : Résultats des AFC effectuées à partir du niveau de catégorisation le plus général (niveau 1) pour $A$ ) les experts et $B$ ) les novices.

La taille des symboles reflète la qualité de la représentation des vins et des descripteurs. 
A : Experts - Niveau de catégorisation II
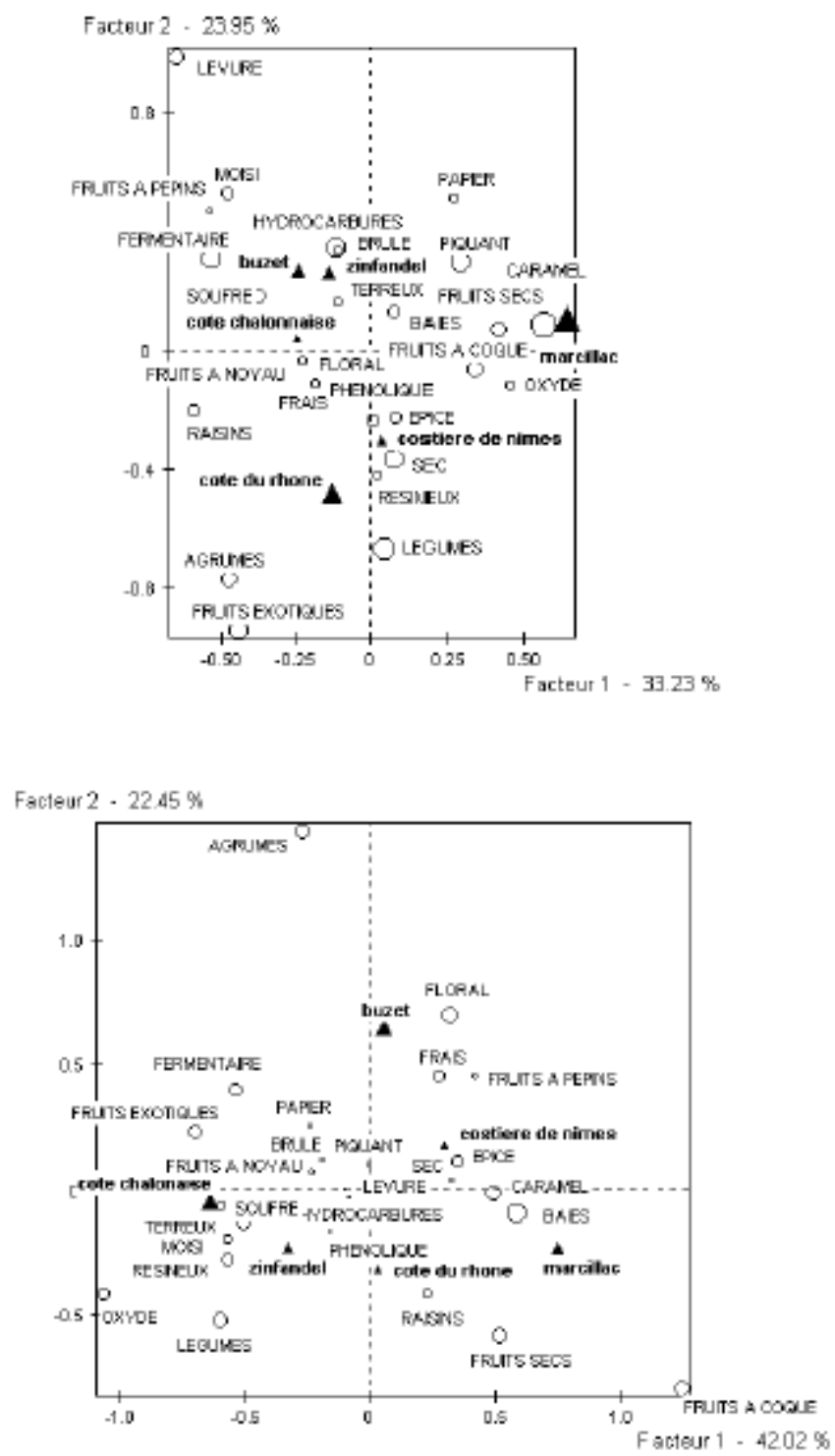

Figure 5 : Résultats des AFC effectuées à partir du niveau de catégorisation intermédiaire (niveau II) pour $A$ ) les experts et $B$ ) les novices.

La taille des symboles reflète la qualité de la représentation des vins et des descripteurs. 


\section{A : Experts - Niveau de catégorisation III}

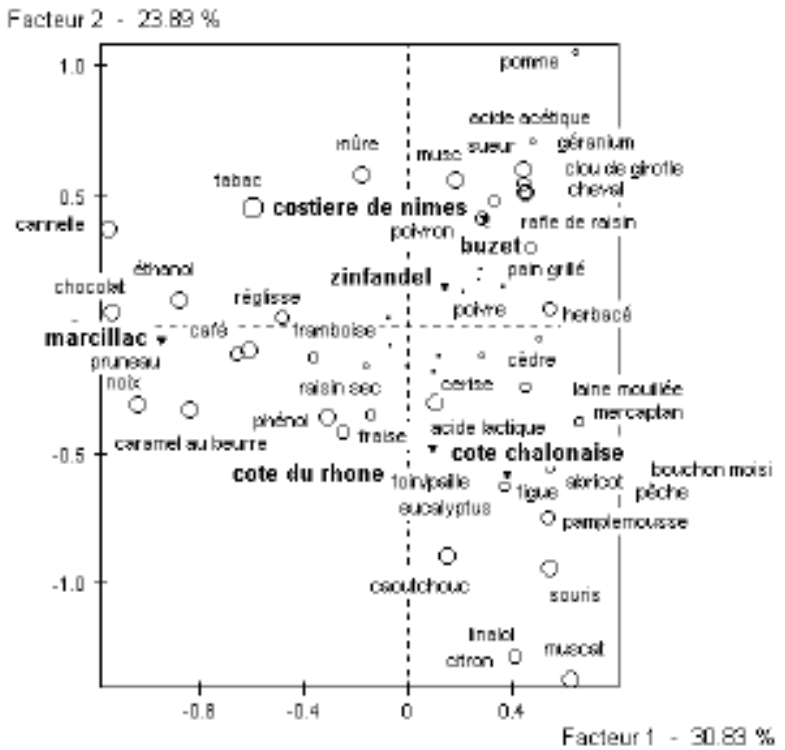

\section{B : Novices - Niveau de catégorisation III}

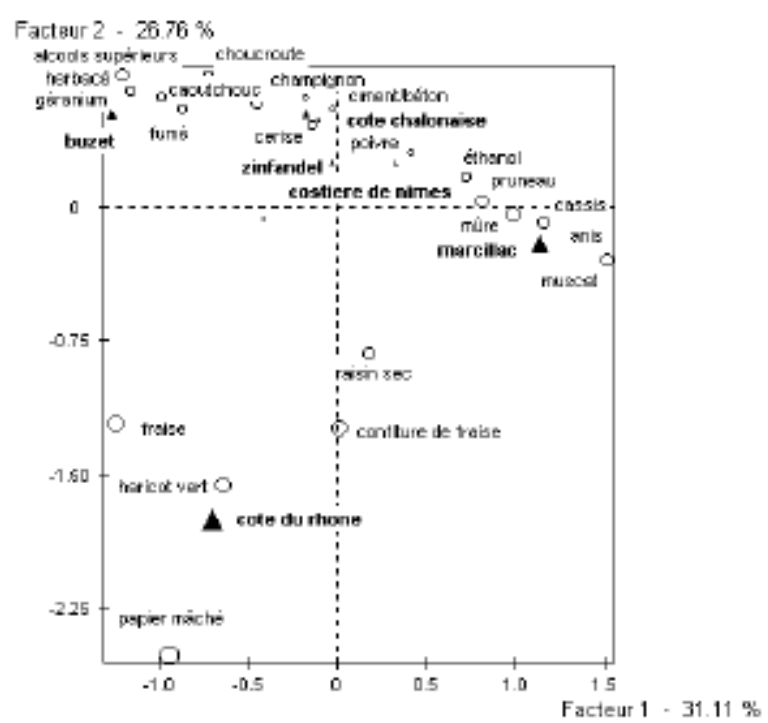

Figure 6 : Résultats des AFC effectuées à partir du niveau de catégorisation intermédiaire (niveau II) pour $A$ ) les experts et $B$ ) les novices.

La taille des symboles reflète la qualité de la représentation des vins et des descripteurs.

Les figures $4 \mathrm{~A}$ et $4 \mathrm{~B}$ représentent, respectivement, les configurations obtenues pour les experts et les novices pour le niveau de catégorisation le plus général (niveau I). Pour les experts comme pour les novices la première dimension de l'AFC oppose le Marcillac qui est perçu comme "fruit à coque » et "caramélisé » par les experts et comme «boisé », «fruité » et «floral » par les novices au Zinfandel et au Côte Chalonnaise qui sont perçus comme «chimique», «microbiologique» et «terreux» par les deux 
groupes de sujets. La seconde dimension, quant à elle, oppose le Costières de Nîmes qui est plutôt "épicé ", «floral " et "végétal » pour les experts et "épicé » pour les novices, au Côte Chalonnaise qui est « chimique » pour les deux groupes de sujets. A coté de ces similitudes, quelques différences peuvent être notées. Ces différences concernent essentiellement le Buzet et le Côte du Rhône. Le Côte du Rhône est perçu comme «microbiologique » et «terreux " par les novices alors qu'il est perçu comme «floral » et «végétal» par les experts. Inversement, le Buzet est perçu comme microbiologique par les experts et végétal par les novices.

17 Les figures $5 \mathrm{~A}$ et $5 \mathrm{~B}$ représentent, respectivement, les configurations obtenues pour les experts et les novices pour le niveau de catégorisation intermédiaire (niveau II). On peut observer une plus grande différence entre la configuration des experts et celle des novices que pour le niveau de catégorisation précédent. Cette plus grande différence est due essentiellement à la modification de la configuration des novices qui maintenant s'écarte de celle des experts. En effet, pour les experts on retrouve l'opposition entre trois groupes de vins qui était observée pour le niveau de catégorisation précédent (i.e.. Marcillac / Costière de Nimes et Côte du Rhône / Buzet, Zinfandel et Côte Chalonnaise). En revanche, pour les novices on observe une modification de la configuration générale des vins. Le Marcillac est toujours opposé au Côte Chalonnaise mais maintenant le point le plus marquant semble être l'opposition entre le Buzet et tous les autres vins. Une distance procrustéenne calculée entre les configurations obtenues pour les deux niveaux de catégorisation confirme la plus grande disparité observée pour les novices $(d=.32)$ par rapport aux experts $(d=.15)$.

Les figures $6 \mathrm{~A}$ et $6 \mathrm{~B}$ représentent, respectivement, les configurations obtenues pour les experts et les novices pour le niveau de catégorisation le plus spécifique (niveau III). La différenciation entre experts et novices augmente encore à ce niveau de catégorisation. Comme précédemment, cette augmentation de la différence entre la configuration des experts et celle des novices s'explique par une modification de la configuration des novices par rapport à l'étape précédente. En effet, à nouveau, la configuration obtenue pour les experts est peu modifiée par rapport à celle obtenue au niveau de catégorisation précédent $(d=.56)$ à l'exception du Côte du Rhône qui maintenant se retrouve plus proche du Côte Chalonnaise que du Costière de Nimes. Par contre, comme précédemment, la distance procrustéenne entre cette configuration et la configuration obtenue au niveau de catégorisation précédente est nettement plus importante pour les novices que celle obtenue pour les experts $(d=1,31)$. On observe maintenant une opposition entre trois vins (Marcillac / Buzet / Côte du Rhône). Les trois autres vins se situent à une position intermédiaire entre le Marcillac et le Buzet. On peut noter également pour les novices une nette séparation du Côte du Rhône par rapport aux autres vins. Ce vin s'est vu attribuer les caractéristiques « haricot vert, fraise, papier mâché, confiture de fraise, et raisin sec ». Une telle séparation n'apparaît pas dans la configuration des experts pour qui le Côte du Rhône est proche de la Côte Chalonnaise. Ces deux vins étant caractérisés, entre autres, par les termes « fraise, phénol, foin/paille, eucalyptus ».

3. Discussion

19 Le niveau d'expertise a-t-il une influence sur la valeur communicative de descriptions de vin? L'analyse des performances obtenues par des sujets experts et des novices lors de la phase d'appariement révèle une légère supériorité des experts sur les novices. Cette supériorité, toutefois, n'apparaît que lorsque les experts ont pour tâche 
d'apparier des descriptions d'experts. Leur performance avec des descriptions de novices ne dépasse effectivement pas le niveau du hasard. Ce résultat suggère donc que la supériorité des experts provient essentiellement de la meilleure qualité de leurs descriptions. En accord avec cette interprétation, les performances des novices s'améliorent quelque peu lorsqu'ils doivent apparier des descriptions d'experts plutôt que des descriptions de novices. Cependant malgré cette amélioration les performances des novices restent significativement inférieures à celles des experts, ce qui indique que la supériorité des experts ne se limite pas à la qualité de leurs descriptions. Chollet et Valentin (2000) proposent deux hypothèses pour expliquer la supériorité des experts dans une tâche de communication. Selon ces auteurs, « soit le vocabulaire utilisé par les experts est plus expressif et permet donc une meilleure description des vins; soit les experts qui ont été formés collectivement à déguster des vins, se comprennent mieux et donc attribuent le même descripteur à la même odeur en utilisant un vocabulaire normé». Ici, les termes n'étaient pas générés par les experts mais seulement sélectionnés sur une liste, et donc la supériorité des experts ne peut s'expliquer par le caractère expressif de leur vocabulaire ni par la pauvreté du vocabulaire à disposition des novices. En revanche, l'analyse de la répartition des termes choisis en fonction de leur niveau de catégorisation montre que les experts utilisent davantage de termes précis que les novices. Des résultats similaires ont également été rapportés par Solomon (1997) dans un travail effectué à partir de la version anglaise de la roue des vins. Il semble donc que ce phénomène ne soit pas lié à une langue particulière et c'est sans doute la précision des experts qui sous-tend leur avantage dans une tâche d'appariement. Cette précision toutefois ne permet pas aux novices d'atteindre des performances supérieures au hasard.

A nouveau deux interprétations peuvent être proposées pour expliquer la différence d'efficacité des termes précis pour les experts et pour les novices. Soit, comme le suggère Chollet et Valentin (2000), un terme doit non seulement être précis pour être efficace, mais il doit être également normé, c'est-à-dire qu'il doit renvoyer à une signification ou référence commune. Dans ce contexte la supériorité des experts s'expliquerait par le fait que lors de leur entraînement les sujets experts apprennent à associer les termes du vin à des références physiques communes (molécules pures ou mélanges de molécules). Soit, la supériorité des experts est due à une meilleure capacité à analyser leurs sensations et à extraire le ou les traits principaux permettant de caractériser un vin ; et cette capacité se traduirait par un choix plus important de termes précis permettant d'attirer l'attention du goûteur sur un aspect caractéristique du vin. Cette dernière hypothèse s'accorde avec la théorie de Gibson (1969) selon laquelle un apprentissage perceptif se traduit par une augmentation de la capacité à extraire des informations d'un environnement lorsqu'il est fréquemment visité. Plus probablement, toutefois, l'avantage des experts est dû à une combinaison de ces deux hypothèses. En effet, contrairement aux experts, les novices ne possèdent que des référents idiosyncrasiques pour les termes précis de la roue des vins; de plus il est difficile pour eux de retrouver les notes correspondant à ces termes dans le mélange odorant complexe que constitue un vin. Par contre, les termes sélectionnés par un expert lui permettrait de focaliser son attention sur quelques caractéristiques prépondérantes $\mathrm{du}$ vin ce qui expliquerait l'amélioration des performances d'appariement. En d'autres termes, un novice confronté à une description d'expert se placerait peut-être dans ce que Bell et Paton (2000) appellent une stratégie de «think 
and sniff » (penser et sentir) plutôt que dans une stratégie de « snif and think » (sentir et penser).

21 En revanche, à un niveau plus général de catégorisation, correspondant à une perception holistique des vins, les experts et les novices semblent se comporter d'une façon similaire. En effet, le calcul des distances procrustéennes entre les configurations issues des AFC réalisées à partir des descriptions des experts et des novices montre que la similitude entre ces descriptions augmente avec le niveau de généralité. Il semble donc que ce qui différencie un expert d'un novice soit précisément l'identification de caractéristiques précises et par là-même l'utilisation de termes précis qui en découle. Cette hypothèse a déjà été avancée dans le domaine du vin (Solomon, 1990 ; Chollet et Valentin, 2000) et de la bière (Chollet et Valentin 2001), mais aussi dans d'autres domaines. Par exemple, Chi et al. (1989) ont montré que des novices utilisent des «traits de surfaces » pour classifier des dinosaures (comme la forme des jambes), alors que les experts basent leur classification sur des traits plus " profonds » (comme leur régime alimentaire) qu'ils auraient appris. Ainsi, experts et novices utilisent le même espace, mais ils diffèrent quand ils le décrivent : les experts décrivent l'espace perceptif commun avec des mots précis et informatifs alors que les novices le décrivent avec des termes globaux et allusifs.

Conclusion

L'analyse des correspondances est rarement utilisée en évaluation sensorielle dans le cadre de la description de produits. La norme AFNOR préconise plutôt l'utilisation de notations sur des échelles suivie d'analyses en composantes principales. L'exemple présenté ici met en évidence l'un des avantages de l'analyse des correspondances dans ce contexte de description : celui d'éviter l'utilisation d'échelles de notation. Basée sur le calcul de distance de khi-deux, l'AFC nécessite seulement l'obtention de tableaux de fréquences de citations de mots. Elle a donc l'avantage de pouvoir être utilisée pour comparer les performances de groupes de sujets n'ayant pas été entrainés à l'utilisation d'échelles de notation. Associée à la technique du calcul de distances procrustéennes, elle permet en outre de quantifier les distances entre les descriptions de ces différents groupes de sujets. Ici elle nous a permit de montrer que la distance entre les descriptions de vins réalisées par un groupe d'experts en vins et celles réalisées par groupe de sujets novices augmentait avec le degré de précision du vocabulaire utilisé.

\section{BIBLIOGRAPHIE}

Benzécri J.P. \& Benzécri F. (1980). Pratique de l'analyse des données. Analyse des correspondances, exposé élémentaire. Paris : Dunod.

Bell G.A. \& Paton J.E (2000). « Verbal-cognitive strategy can influence odor judgment », AromaChology Review $9: 3-9$.

Chi M.T.H., Bassok M., Lewis M.W., Reimann P. \& Glaser R. (1989). « Self explanations : How students study and use examples in learning to solve problems », Cognitive Science 13 : 145-182. 
Chollet S. \& Valentin D. (2000). « Le degré d'expertise a-t-il une influence sur la perception olfactive ? Quelques éléments de réponse dans le domaine du vin », L'Année Psychologique 100 : 11-36.

Chollet S. \& Valentin, D. (2001). « Impact of training on beer flavor perception and description : are trained and untrained subjects really different? ", Journal of Sensory Studies 16 : 601-618.

Gawel R. (1997). « The use of language by trained and untrained experienced wine tasters ", Journal of Sensory Studies $12: 267-284$.

Gibson E.J. (1969). Principles of perceptual learning and development. Englewood Cliffs, NJ : Prentice Hall.

Gower J.C. (1971). « Statistical methods of comparing different multivariate analysis of the same data », in F.R. Hodson, D. Kendall \& P. Tautu (eds.) Mathematics in the archeological and historical sciences. Edinburgh : Edinburgh University Press, 138-149.

Lawless H.T. (1984). «Flavor description of white wine by "expert" and nonexpert wine consumers ", Journal of Food Science $49: 120-123$.

Lehrer A. (1975). « Talking about wine », Language 51 : 901-923.

Peynaud E. \& Blouin, J. (1996). Le goût du vin. Paris : Dunod.

Solomon G.E.A. (1990). « Psychology of novice and expert wine talk », American Journal of Psychology 103 : 495-517.

Solomon G.E.A. (1997). « Conceptual change and wine expertise », The Journal of the Learning Sciences $6: 41-60$.

\section{NOTES}

1. Les auteurs tiennent à remercier Marie Pichon pour son aide dans la passation des expériences.

\section{RÉSUMÉS}

Un expert en vin perçoit-il et décrit-il l'odeur d'un vin de façon plus analytique qu'un novice? Pour répondre à cette question, nous avons demandé à un groupe d'experts et un groupe de novices de décrire des vins à l'aide d'une liste de termes organisée en trois niveaux de catégorisation allant du plus général au plus spécifique. Nous avons ensuite demandé aux mêmes sujets d'apparier les descriptions réalisées par une autre personne avec les vins correspondants. Les résultats montrent que les descriptions effectuées par les experts sont plus précises et conduisent à de meilleures performances d'appariement que celles effectuées par les novices. De plus, la distance entre les descriptions d'experts et celles de novices augmente avec le niveau de précision des termes.

The words of wine

Do wine experts perceive and describe wines more analytically than novices? To answer this 
question we asked a group of wine experts and a group of novices to describe wines with a list of terms organized hierarchically in three levels (from general to specific). Then we asked the same subjects to find the wine described by other subjects. We found that experts are more precise in their description and that it is easier for subjects to identify a wine described by an expert than to identify a wine described by a novice. We also found that experts and novices differ most when they use specific terms.

INDEX

Mots-clés : descripteurs olfactifs, expertise, vin

\section{AUTEURS}

DOMINIQUE VALENTIN

CESG, Université de Dijon

SYLVIE CHOLLET

ISA, Université Catholique de Lille

HERVÉ ABDI

The University of Texas at Dallas 